\title{
Effect of Stick - Slip Phenomena between Human Skin and UHMW Polyethylene
}

\author{
Emad Kamil Hussein*, Kussay Ahmed Subhi and Tayser Sumer Gaaz \\ Al-Mussaib Technical College TCM, Al-Furat Al-Awsat Technical University ATU, \\ P.O. Box 51006, Al-Mussaib, Babil, Iraq
}

\begin{abstract}
The present paper investigates experimentally effect of applied load and different velocity on the coefficient of friction between two interacting surfaces (human skin and Ultra-highmolecular-weight polyethylene (UHMW- polyethylene) at static and dynamic friction. It is possible to conclude specific point based on the above practical part and frictional analysis of this investigation as the most important mechanical phenomenon was creep has been observed a stick time interval where the static friction force is significantly increased during this stroke. The analytical model for stick-slip of skin and UHMWPE is proposed. The difference between static and kinetic friction defines the amplitude of stick-slip phenomena. The contact pressure, the sliding velocity, and rigidity of system determine the stability conditions of the movement between skin and UHMWPE. Experiments were carried out by developing a device (friction measurement). Variations of friction coefficient during the time at different normal load 4.6 and $9.2 \mathrm{~N}$ and low sliding velocity 4, 5, 6 and $7 \mathrm{~mm} / \mathrm{min}$ were experimentally investigated. The results showed that the friction coefficient varied with the normal load and low sliding velocity. At static friction, the coefficient of friction decreased when the time increases, whereas, at dynamic friction, the coefficient of friction decreased when the time increased at normal load 4.6 and $9.2 \mathrm{~N}$.
\end{abstract}

ARTICLE INFO

Article history:

Received: 02 December 2020

Accepted: 16 February 2021

Published: 31 July 2021

DOI: https://doi.org/10.47836/pjst.29.3.06

$\overline{\text { E-mail addresses: }}$

SHARAF.MOHAREB@gmail.com; emad_kamil72@atu.edu.iq

(Emad Kamil Hussein)

Kussaysubhi@atu.edu.iq (Kussay Ahmed Subhi)

Tayser1Gaaz@gmail.com (Tayser Sumer Gaaz)

* Corresponding author
Keywords: Friction dynamic, friction static, human skin, stick-slip

\section{INTRODUCTION}

Human skin is considered as a complex multilayers tissue with a viscoelastic (nonlinear) mechanical property, so that means it will exhibit a specific direct contact with other materials, especially in case of 
frictional behavior considering contribution of each layer. Researchers are stimulated to investigate such kind of human skin friction.

Crowther et al. (2004) investigated a direct contact effect of applying normal load and probe frictional sliding speed on the biotribological features of skin and analyzing such specific frictional behavior and mechanism. A multi-specimen friction rig has been employed to conduct the experimental part of this investigation, where the normal load varies from starting value of 0.1 up to $0.9 \mathrm{~N}$. So, because of this increment, both of normal displacement and the associated coefficient of friction are increased, formation of two frictional components adhesion and deformation was behind of this increment. On the other side, creation of stick-slip phenomenon was the main factor that contributes to rise the value of the induced coefficient of friction as the sliding speed from initial value of 0.5 millimeter per second up to a final value of 4 millimeters per second. In addition, based on hysteresis friction principle, Tang et al. (2008) presented a detailed logic explanation of the expected mutual influence of normal load and sliding speed on frictional behavior of skin.

Chatelet et al. (2008) reviewed a recent factor that was closely related to human skin frictional tribology including experimental examination for human skin frictional factors based on many zones in human body. First factor under investigation was direct contact skin pressure induced under wide range of boundary conditions and measurements. Derler and Rotaru (2013) concluded that almost all previous researches were explaining about the two frictional components, adhesion and deformation. Furthermore, it is seen that dry skin gives a specific value of coefficient of friction ranging around 0.5 but the situation is completely different in case of moist skin where the same coefficient under the same loading conditions is fluctuating above-leading to decrease contact pressure significantly.

Derler and Rotaru (2013) developed a special device that is used to measure complementary features to the viscoelastic properties of human skin by employing a dynamic indenter, so the two important parameters were human skin stiffness exactly like this one for an elastic element, spring, and the damping coefficient like the vicious element, dashpot, where these two mechanical parameters are completing the other essential tribological properties of human skin. Results showed that complex modulus measured by this modified dynamic indentation technique was about 7.2 KPa for aged volunteer and 10.7 for younger ones, hence, the main reason that causes such variation in the measured values was the nature of human skin as a function of time and the associated semi degeneration of some specific layers with human skin as time goes on.

Dong et al. (2017a) have developed a special tribe-acoustical probe that has been used to measure a friction noise via recording the generated sound of a pressure level in case of direct dynamic contact between two mating surfaces, the first one is representing an artificial finger when rubbing on various surfaces. An attached microphone has been installed to the modified sensing system to soundtrack different levels of the generated 
sounds and then comparing these acquired sounds to precisely assess smoothness in all rubbing cases. Final observations showed that friction noise gives an excellent indication of softness and smoothness in such tribological friction cases.

Dong et al. (2017b) studied mechanical and viscoelastic properties of human skin in vivo as a first step to assess the direct and side effects of cosmetical and medical products on human skin. Boyer et al. (2007) developed a non-invasive rig that is used to examine viscoelastic properties of human skin in vivo based on direct dynamic indentation. Obtained findings showed the Kelvin-Voigt model was well expressing the mechanical behaviors of such kind of skin with dynamic indentation effect. In addition, a mean value of stiffness and damping coefficient were also considered into account to compare skin viscoelastic features. Final experimental outcomes showed an accurate value of the measured factors, which surely means it is possible to use this device as a viscoelastic Tribometer in future work.

Mulliah et al. (2004) examined some definite parameters including finger hydro-lipid skin film, overall shape, precise surface roughness, and rigidity for both men and women on friction conditions in case of direct rubbing between these fingers and other selected surfaces. Four surfaces were considered in this research, two of them are real and the remaining is virtual. A developed stimulator named STIMTAC has been employed to achieve goals for both cases of men's and women's fingers skin. A significant dissimilarity between men's and women's finger surface topography has been also observed and this fact is due to men's finger skin are rougher than for women's fingers.

Perfilyev et al. (2013) conducted an experimental investigation about the stick-slip phenomenon in case of friction between the index finger pad in direct sliding on wet surface and smooth surface glass taking into consideration the amount of the applied force and the associated sliding velocity by engaging a tri-axial force plate rig. The attained coefficient of friction during the stick-slip phase was $30 \%$ lower than for the case of stationary sliding, plus final finding exhibits that during the stick-slip period coefficient of friction varies by about $\pm 25 \%$ around the mean value, hence it is accepted to consider the achieved results as a basis of artificial skin in mechanical manipulators especially in the interaction between precision riggings and soft tissues.

Terrand-Jeanne and Martins (2016) created a comparison between the obtained elastic constant (stiffness) for collagen and elastin within human skin with other same investigations in previous publications. The basis of this paper was about the precise examination of viscoelastic properties of human skin via determining the variation of stress-strain interaction, so as usual the induced stiffness was measured directly from the elastic stress-strain diagram, meanwhile, the collagen fibril length was acquired from the mathematical slope of a viscous stress-strain diagram. Finally, stiffness for collagen was about $4.4 \mathrm{GPa}$ and for elastin $4 \mathrm{MPa}$, and this is due to alterations in the collagen forms and the associated natures in human skin and tendon which may significantly affect such specific viscoelastic properties. 
Tu and Fort (2004) have suggested a parametric finite element contact homogenization simulation procedure to predict the expected effect of the skin topography nature including microstructure on the measured response of human skin friction, so depending on simulation of sliding of different indentation probes on human skin. Results illustrate the significant effect of the real complex geometry of human skin cross-sectional microstructure on the induced deformation component of such kind of specific friction and hence the complicity leads to increase this essential component plus the generated global friction coefficient was differing from the local one where the global was lower than the other coefficient.

Wee et al. (2001) studied the formation of the stick-slip phenomenon in two cases, the first one was in the case of low sliding speed of the probe on the opposite surface, meanwhile, the second case was at a very low sliding speed. It is considered that the induced static coefficient of friction ( $\left.\mu \_s\right)$ between the two mating surfaces must be greater than the kinetic coefficient of friction $\left(\mu \_\mathrm{k}\right)$ conclusion was stated that using bearing will significantly decrease the effect of stick-slip occurrence. In this research, the Effect of StickSlip Phenomena between Human Skin and UHMW Polyethylene has been investigated experimentally. Velocity and force were the main indicators of the analysis of the skin characterization.

\section{MATERIALS AND METHODS}

The first part device called (stick-slip device) manufactured in Romania related to the mechanical parts whereas the electronic parts were designed and instated on the device by the authors to measures the skin friction at different velocities as shown in Figure 1. The main parts of this device are the electric motor, gearbox, tangential sensor, and load cell sensor modified by the authors (Figures 2 and 3 ).

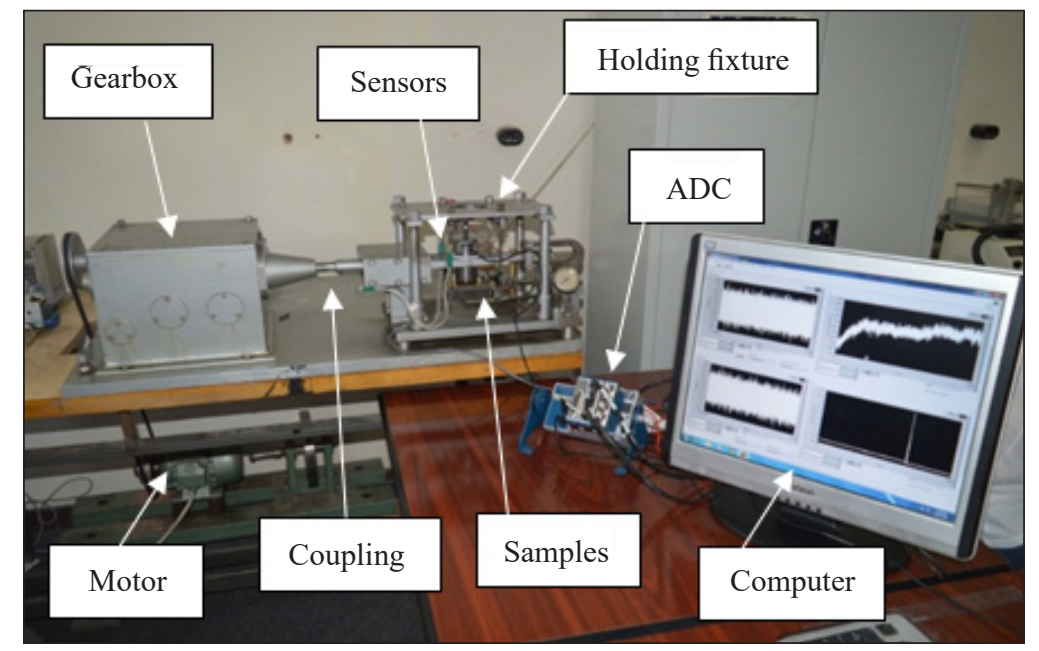

Figure 1. The developed rig 


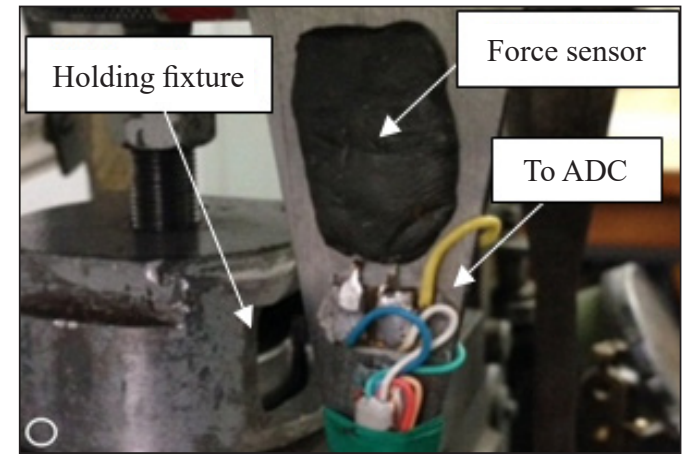

Figure 2. The attached tangential force sensor

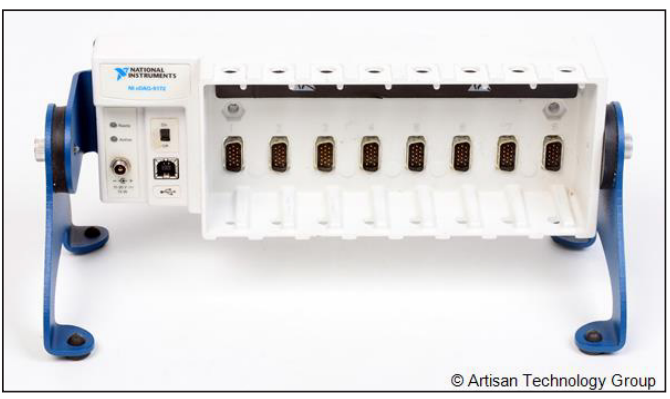

Figure 4. The employed ADC

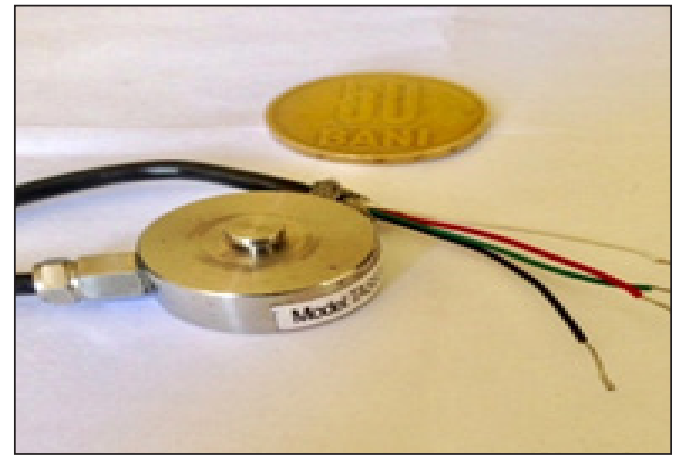

Figure 3. The employed load cell sensor

In case of requiring a directly applied load, then it is usually depending on the so-called load cell, and this kind of sensors can transform applied load or pressure up to $10 \mathrm{Kg}$ into an electrical pulse, in other words, the load cell measures the variance in electrical resistance based on the amount of the applied load so that means the electrical resistance variation is directly proportional to the associated applied load, force or pressure. In many specific cases of friction, it is best to use disk load cells, that are having a suitable design and flexible handling with the frictional case under consideration than the bar-style cells. Load cells are manufactured from alloy steel and are having four strain gages, four electric resistances that are forming the Wheatstone electrical bridge, which is used to measure unknown value electric resistance by creating a balance in the other two terminals (legs) of the bridge. There are many types of such electric bridges and the common one is having $(220 * 13 * 1000) \mathrm{mm}$ dimensions.

Regarding stick-slip the device, a Converter was used to transfer the signal from analog to digital was connected between the sensors and computer model (NI cDAQ-917) as shown in Figure 4. The all-force measurement data was used for the data analyses for which specific programs were developed using the software LabVIEW. The tip used in this study made from UMHW polyethylene with a dimension (diameter is $7 \mathrm{~mm}$; section area is $38.5 \mathrm{~mm} 2$ ).

\section{Conditions}

In this study, the ambient conditions were as follows, room temperature $25^{\circ} \mathrm{C}$ and approximate humidity of about (38-45) \%, The experiment was repeated at less three times and the average was considered. Before the examinations, the prepared skin was 
well prepared to fit the local laboratory boundary conditions for at least 10 minutes, the test sites were marked to ensure repeatability of the data. The index finger skin has been very well cleaned by using the Ethanol alcohol and then washed with sterilized water. Before starting the investigation as much as other examinations plan, at the same moment the indenter tip UHMW polyethylene material also cleaned with ethanol and water. All friction measurements were carried out by the same subject (male, 49 years), using the index finger of the dominant.

\section{Device for Friction Study at Low Slipping Velocity}

- The employed rig has been modified by attaching a suitable load (force) sensor to be connected to a specific software program named LabVIEW via a desktop computer and then adjusted and normalized by checking various load values starting from zero up to 1800 grams with step increment of 150 grams per each step, where the first experimental step was setting the nominated skin without any tension or compression stresses as shown in Figure 1.

- $\quad$ ASTM D3108 Standard Test Method for Coefficient of Friction, Yarn to Solid Material has been employed to carry out the results. The author observed the new device has a higher accuracy with a lower price compared with the UMT device. However, before the experimental with the second device (stick-slip device) starting there are several parameters were adjusted as follows:

- Time duration for each frictional test was about 60 seconds, and the total number of repetitions were 48 single frictional tests.

- Maximum sliding distance of the finger with UHMWPE was between 4-7 mm.

- Range of normal forces is 4.6 up to $9.2 \mathrm{~N}$.

- Sliding velocities were $4,5,6$ and $7 \mathrm{~mm} / \mathrm{min}$.

First Step. Applied normal force $9.2 \mathrm{~N}$ at different velocities $(4,5,6,7 \mathrm{~mm} / \mathrm{min})$ as shown in Figure 5 the stick-slip phenomena appeared at the time $(0-60 \mathrm{sec})$, for velocity $4 \mathrm{~mm} /$ min the stick-slip is very clear compared to other velocities.

Second Step. Applied normal force $4.6 \mathrm{~N}$ at different velocities $(4,5,6,7 \mathrm{~mm} / \mathrm{min})$ as shown in Figure 6 the stick-slip phenomena appeared at the time $(0-40 \mathrm{sec})$, for velocity $5 \mathrm{~mm} / \mathrm{min}$ the stick-slip is clear compared to other velocities.

\section{RESULTS AND DISCUSSION}

The final practical and experimental results of this investigation have been acquired from the employed modified devices that are related to clarifying the outcome of frictional behavior of human skin at various boundary conditions, so these gained results are listed below. 


\section{Stick-slip Device}

The stick-slip behavior of the index finger sliding and UHMW polyethylene was examined as a function of normal applied force and the associated sliding velocity in this specific kind of frictional measurement by using (stick-slip device). The friction coefficients during stick-slip were $(\min =0.27, \max =0.32)$, which clearly gained to be typical of about $25.6 \%$ lower than those of stationary sliding test $(\min =0.36, \max =0.43$ ) as shown in Figure 5. Stick-slip frictional tests noted took place throughout the entire frictional experiments or alternated with the stationary sliding frictional phases.

It was observed at the applied load of $9.2 \mathrm{~N}$ decrease the coefficient of friction with the increase in the velocity of sliding friction significantly compared to when the load of 4.6 Newton due to human skin shows a complicated surface topography and, viscoelastic properties (non-linear behavior), resulting in a very complex mechanical direct contact conditions and specific frictional behavior. Physiological responses including sweating will additionally complicate this mutual contact mechanics by interactively varying the caused interface between the human skin and counter-surface at the region under consideration. Also, detected for a normal applied force of about $9.2 \mathrm{~N}$ the full detailed breakdown displays that the periods of formation of sticking were a direct factor of around two greater than the periods of slipping. In contrast, observed for a normal force of $4.6 \mathrm{~N}$ the detailed analysis shows that the periods of sticking were a factor of around 3 times longer than that of the periods of slipping. As shown in Figures 5 and 6 when drawing zoom.

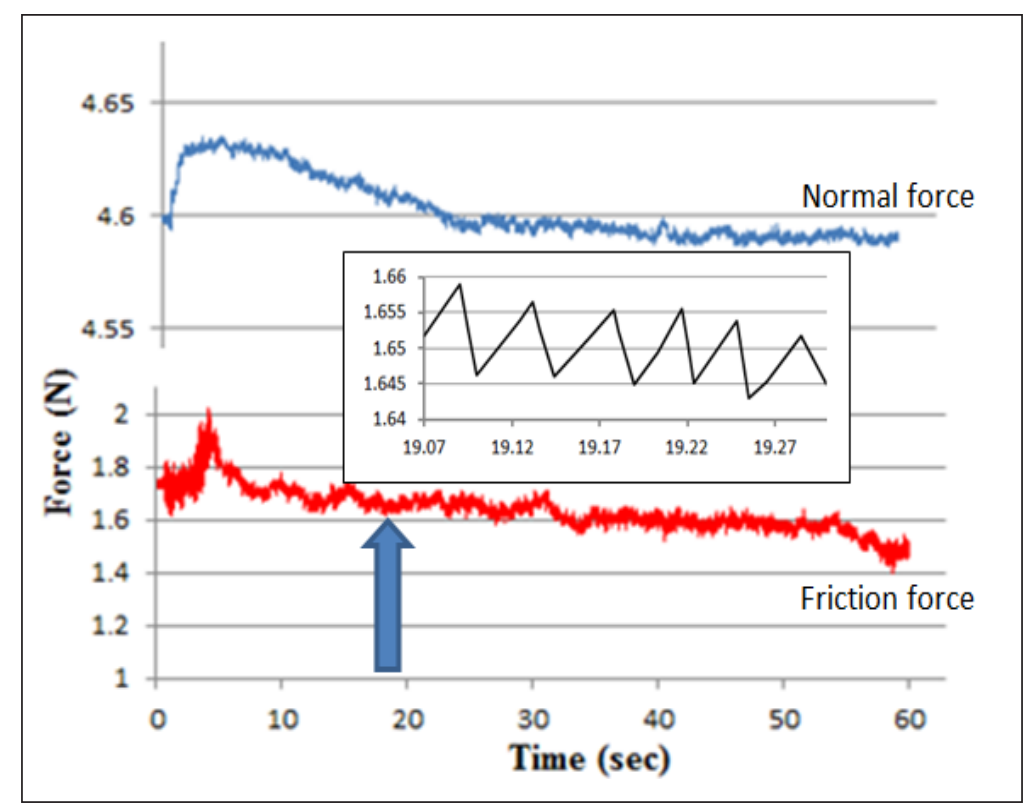

Figure 5. Normal force against test time duration, at load of $4.6 \mathrm{~N}$ 


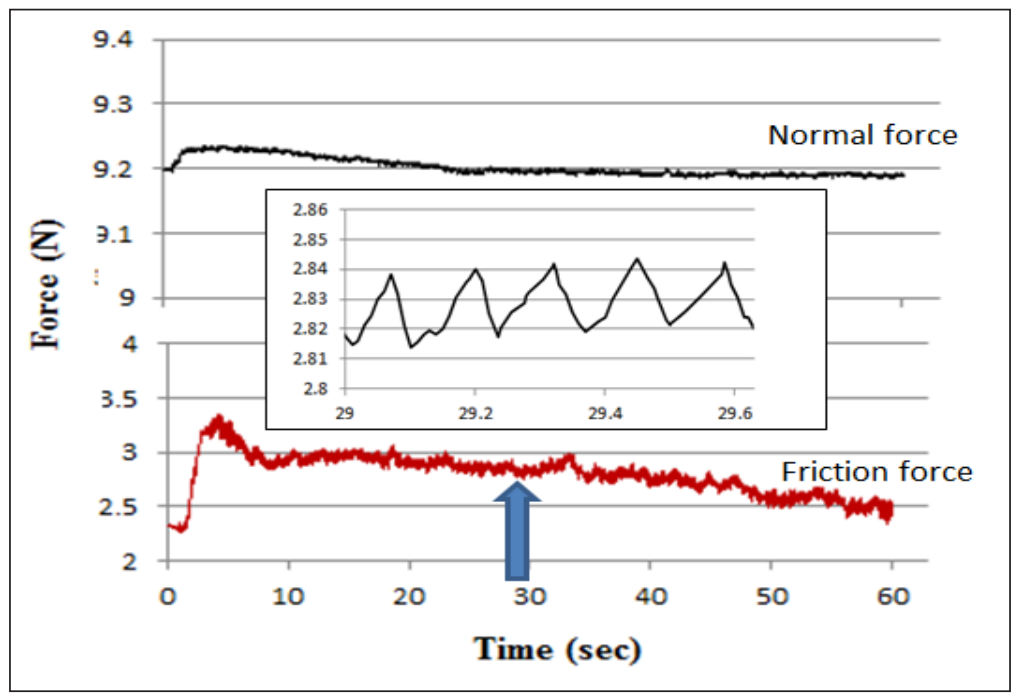

Figure 6. Normal force vs test time, at load $9.2 \mathrm{~N}$

\section{Effect of Sliding Velocity and Friction Mechanism}

The sliding velocity and friction mechanism were done by logically increasing sliding velocity from $0.3 \mathrm{~mm} / \mathrm{min}$ to $7 \mathrm{~mm} / \mathrm{min}$. As sliding velocity increases friction coefficient decreases and hence through assessment fitting the correlation coefficient reaches up to 0.96 and 0.91 at normal load 4.6 N and 9.2 N respectively as shown in Figure 7. During the sliding hypothesis, almost all the sliding energy is released when the applied stress is completely removed from the contact region skin. The hysteretic skin friction was relatively small in amount because of lost a small amount of energy, whereas, at higher values of sliding speed, the local deformation rate on the employed skin is also increased and the obtained deformation is so difficult to local recover instantaneously.

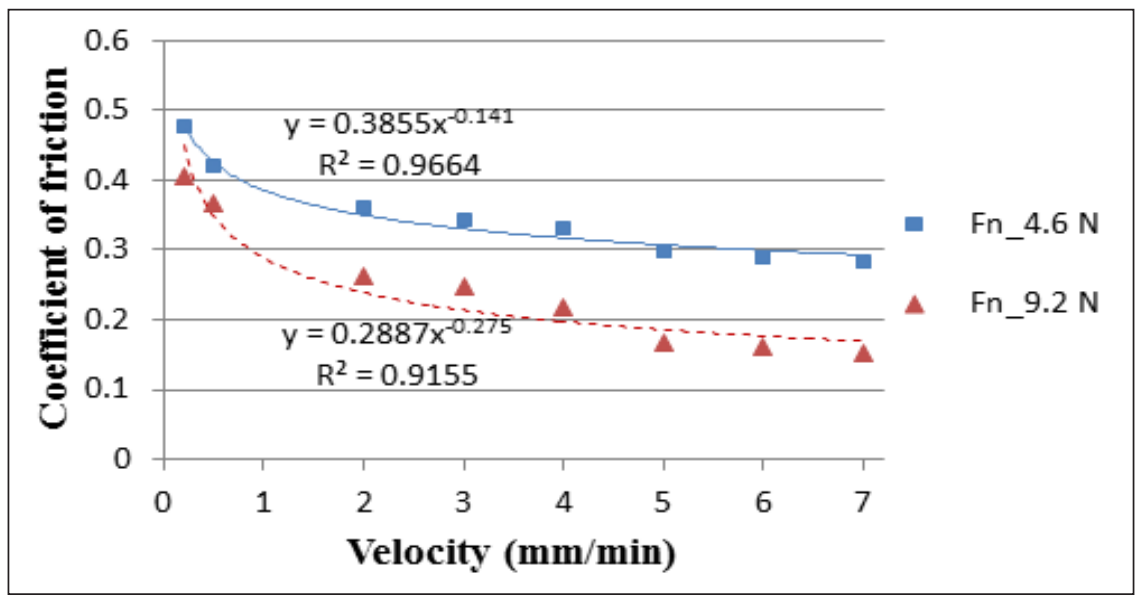

Figure 7. Coefficient of friction vs velocity 


\section{Effect of Normal Load and its Mechanism}

The examinations of applying normal loads were conducted by the gradual increase of the applied normal loads from an initial value of $2.3 \mathrm{~N}$ up to $19.3 \mathrm{~N}$. The obtained results with a fitted curve of the gained coefficient of friction against the applied normal loads are shown in Figure 8. Figure 8 shows the results of the load based on the practically measured coefficients of friction. Here, the mean friction coefficient was mathematically calculated for the principal sliding phases and graphically plotted against the induced mean normal force. The two sole factors that are leading to such relatively small values of the standard deviations between (0.0045-0.0161) were the expected variations in the total friction force during the stick-slip phase as well as variations in the direction normal force applied by the external weight during each individual frictional experiment. A fit to the gained results by using the so-called Power-law function when use different velocity gives Equation 1 and 2:

$$
\begin{aligned}
& \mu\left(F_{n}\right)=0.677 F_{n}^{-0.29} \\
& \mu\left(F_{n}\right)=0.695 F_{n}^{-0.441}
\end{aligned}
$$

The correlation coefficient reaches 0.973 and 0.948 , respectively as the coefficient of friction is proportional to the applied normal load, where $\mu$ denotes the friction coefficient and $F_{n}$ the normal force.

To accurately estimate the rising direct contact pressure at the specified skin region, the contact area of the human index finger was measured as a function of the applied normal force. The specified area of the finger direct contact area with UMHW polyethylene area measured in $\mathrm{mm}^{2}$ on the normal force $\mathrm{F}_{\mathrm{n}}(\mathrm{N})$ approximately associated to the function as shown in the following Equation 3:

$$
A\left(F_{n}\right)=0.275 F_{n}^{-0.195}
$$

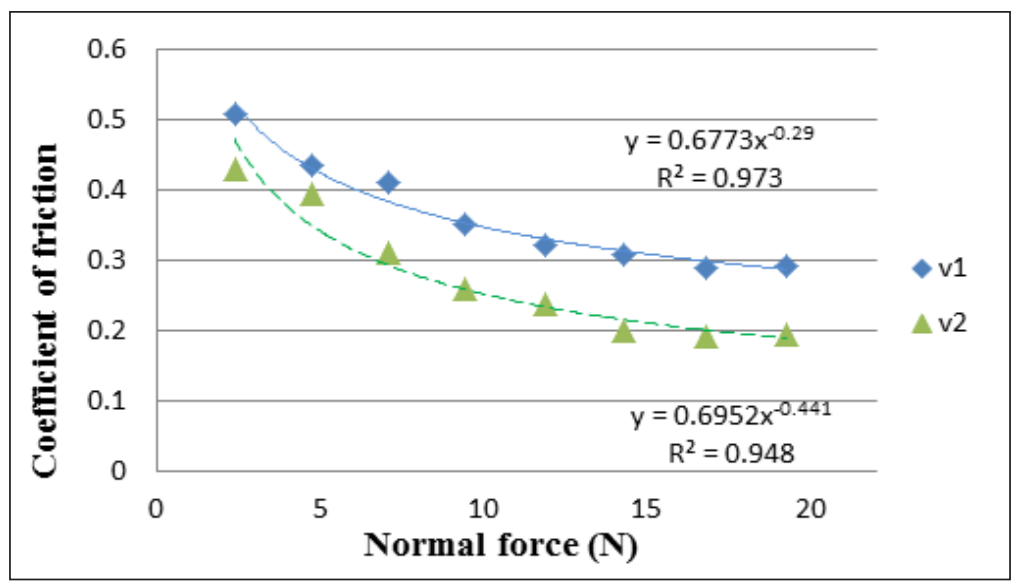

Figure 8. Coefficient of friction against normal load 
Figure 9 presents the pressure-dependence of the friction coefficients. It is clear to be distinguished that the real contact area between the index finger and UMHW polyethylene on the infinitesimal level is considerably smaller than that of the apparent contact area, causing to relatively higher real contact pressures in the definite skin contact zones, therefore, it can be supposed that the micro-mechanical behavior of skin is characterized by a higher modulus of elasticity.

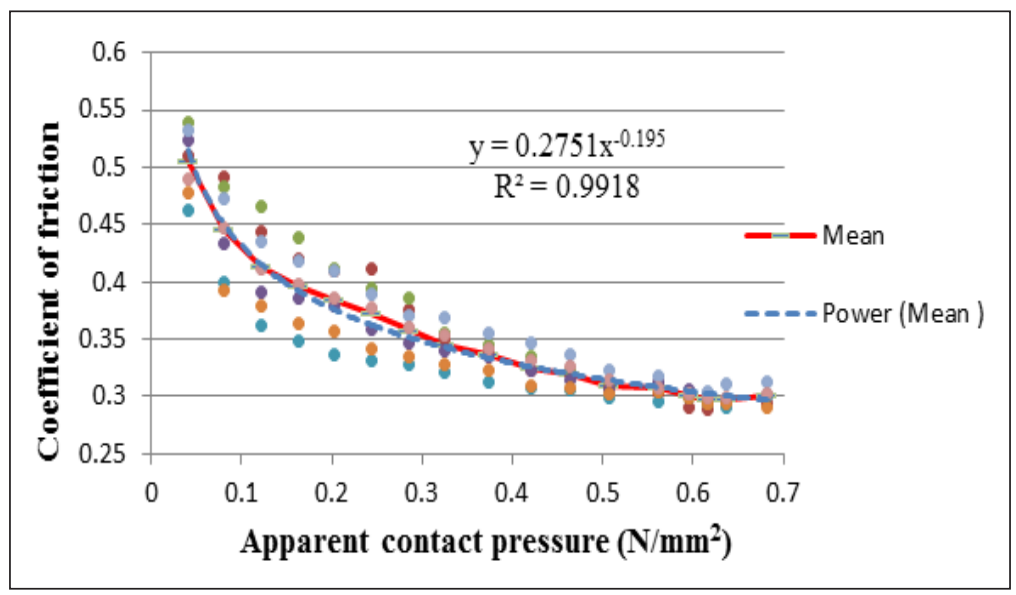

Figure 9. Coefficient of friction as a function of contact pressure

\section{CONCLUSION}

- It is possible to conclude specific point based on the above practical part and frictional analysis of this investigation as:

- One of the most important mechanical phenomenon was creep has been observed a stick time interval where the static friction force is significantly increased during this stroke, The analytical model for stick-slip of skin and UHMWPE is proposed.

- The difference between static and kinetic friction defines the amplitude of stickslip phenomena. The contact pressure, the sliding velocity, and rigidity of system determine the stability conditions of the movement between skin and UHMWPE.

- The normal applied load effects the frictional behavior of human skin friction and the coefficient of friction shows utterly load dependence.

- When the normal load increases from $2.3 \mathrm{~N}$ to $19.3 \mathrm{~N}$, the friction coefficient of skin decrease.

- The sliding speed influences the frictional behavior of human index finger skin when the adjusted sliding speed is increasing from initial value of $0.3 \mathrm{~mm} / \mathrm{min}$ up to $7 \mathrm{~mm} / \mathrm{min}$, and the coefficient of friction decreases, plus the "stick-slip" phenomenon appears. 
- Obtained results for the stick-slip friction tests with the human finger might be valuable in connection with the improvement of the prosthetic socket and for the accurate control of the formation of stick-slip friction between of the skin and the inner layer of the socket.

\section{ACKNOWLEDGEMENT}

The present work was undertaken under the support of the State Company for Fabrics Industries (SCFI), for the preparing and providing of all fabrics, and funded by Al-Furat Al-Awsat Technical University (ATU), Iraq. Also, many thanks for the all amputee persons. The author acknowledges the constant support encouragement of Professor Dr. Eng. Andrei Tudor, Politehinca Univ. of Bucharest, Romania, and Prof. Dr. Eng. Muhsin J. J. Head of P\&O Eng. Dept. at College of Engineering, Nahrain University, Baghdad, Iraq.

\section{REFERENCES}

Boyer, G., Zahouani, H., Le Bot, A., \& Laquieze, L. (2007). In vivo characterization of viscoelastic properties of human skin using dynamic micro-indentation. In 2007 29th Annual International Conference of the IEEE Engineering in Medicine and Biology Society (pp. 4584-4587). IEEE Conference Publication. https://doi.org/10.1109/IEMBS.2007.4353360

Chatelet, E., Michon, G., Manin, L., \& Jacquet, G. (2008). Stick/slip phenomena in dynamics: Choice of contact model. Numerical predictions \& experiments. Mechanism and Machine Theory, 43(10), 12111224. https://doi.org/10.1016/j.mechmachtheory.2007.11.001

Crowther, A., Zhang, N., Liu, D. K., \& Jeyakumaran, J. K. (2004). Analysis and simulation of clutch engagement judder and stick-slip in automotive powertrain systems. Proceedings of the Institution of Mechanical Engineers, Part D: Journal of Automobile Engineering, 218(12), 1427-1446.) https://doi. org/10.1243/0954407042707731

Derler, S., \& Rotaru, G. M. (2013). Stick-slip phenomena in the friction of human skin. Wear, 301(1-2), 324329. https://doi.org/10.1016/j.wear.2012.11.030

Dong, C., Shi, L., Li, L., Bai, X., Yuan, C., \& Tian, Y. (2017a). Stick-slip behaviours of water lubrication polymer materials under low speed conditions. Tribology International, 106, 55-61. https://doi.org/10.1016/j. triboint.2016.10.027

Dong, C., Yuan, C., Bai, X., Qin, H., \& Yan, X. (2017b). Investigating relationship between deformation behaviours and stick-slip phenomena of polymer material. Wear, 376, 1333-1338.|https://doi.org/10.1016/j. wear.2017.01.061

Mulliah, D., Kenny, S. D., \& Smith, R. (2004). Modeling of stick-slip phenomena using molecular dynamics. Physical Review B, 69(20), Article 205407. https://doi.org/10.1103/PhysRevB.69.205407

Perfilyev, V., Moshkovich, A., Lapsker, I., Laikhtman, A., \& Rapoport, L. (2013). The effect of vanadium content and temperature on stick-slip phenomena under friction of $\mathrm{CrV}$ (x) N coatings. Wear, 307(1-2), 44-51. https://doi.org/10.1016/j.wear.2013.08.012 
Tang, W., Ge, S. R., Zhu, H., Cao, X. C., \& Li, N. (2008). The influence of normal load and sliding speed on frictional properties of skin. Journal of Bionic Engineering, 5(1), 33-38. https://doi.org/10.1016/S16726529(08)60004-9

Terrand-Jeanne, A., \& Martins, V. D. S. (2016). Modeling's approaches for Stick-Slip phenomena in drilling. IFAC-PapersOnLine, 49(8), 118-123.' https://doi.org/10.1016/j.ifacol.2016.07.428

Tu, C. F., \& Fort, T. (2004). A study of fiber-capstan friction. 2. Stick-slip phenomena. Tribology International, 37(9), 711-719.' https://doi.org/10.1016/j.triboint.2004.02.009

Wee, H., Kim, Y. Y., Jung, H., \& Lee, G. N. (2001). Nonlinear rate-dependent stick-slip phenomena: Modeling and parameter estimation. International Journal of Solids and Structures, 38(8), 1415-1431.] https://doi. org/10.1016/S0020-7683(00)00058-5 